\title{
Comparison of the Serological Reactivity of Lipopolysaccharides from Japanese and Western Strains of Helicobacter pylori to Sera from $H$. pylori-Positive Humans
}

\author{
Ken-ichi Amano, ${ }^{1}$ Shin-ichi Yokota, ${ }^{2}$ and Mario A. Monteiro ${ }^{3}$ \\ ${ }^{1}$ Bioscience Education and Research Center, Akita University, 1-1-1, Hondo, Akita 010-8543, Japan \\ ${ }^{2}$ Department of Microbiology, Sapporo Medical University School of Medicine, South-1, West-17, Chuo-ku, \\ Sapporo 060-8556, Japan \\ ${ }^{3}$ Department of Chemistry, University of Guelph, Guelph, ON, Canada N1G 2W1
}

Correspondence should be addressed to Shin-ichi Yokota, syokota@sapmed.ac.jp

Received 28 April 2012; Accepted 11 June 2012

Academic Editors: H. I. Atabay, C. K. Cote, and D. Rodriguez-Lazaro

Copyright ( $) 2012$ Ken-ichi Amano et al. This is an open access article distributed under the Creative Commons Attribution License, which permits unrestricted use, distribution, and reproduction in any medium, provided the original work is properly cited.

We compared the serological reactivity of lipopolysaccharides (LPS) isolated from Japanese and Western strains of Helicobacter pylori against anti-Lewis antigen monoclonal antibodies and $H$. pylori-positive Japanese sera. The two LPS from Western strains (26695 and O:2) did not react with any sera from Japanese patients, while all LPS from Japanese strains and the Sydney strain reacted with these sera. We propose that LPS of all Japanese smooth strains share either one of two epitopes, which are termed highly antigenic and weakly antigenic epitopes, present in the O-polysaccharide portion, and these epitopes are independent the Lewis antigens. The present findings indicated that the two Western strains lacked the two epitopes, which are shared by all Japanese strains.

\section{Introduction}

Helicobacter pylori is a gram-negative and microaerophilic bacterium that is recognized as a major cause of chronic gastritis, peptic ulcer, and gastric cancer $[1,2]$. The chemistry and biology of $H$. pylori lipopolysaccharides (LPS) have been extensively studied. Aspinall et al. [3] and Monteiro et al. [4] determined the structures of the O-polysaccharides of H. pylori LPS and found them to be the same as the Lewis $\mathrm{X}\left(\mathrm{Le}^{\mathrm{x}}\right)$ and Lewis $\mathrm{Y}\left(\mathrm{Le}^{\mathrm{y}}\right)$ determinants of human cellsurface glycoconjugates. Appelmelk et al. [5] suggested that the mimicry of Lewis antigens by this bacterium raised titers of autoantibodies to Lewis antigens in infected individuals. However, we find no significant titers of anti-Lewis antigen antibodies in the sera of $H$. pylori-positive humans [6]. On the other hand, we have observed that all $H$. pylori smoothtype LPS possess either one of two antigenic epitopes (the highly antigenic and the weakly antigenic epitopes) in their polysaccharide regions [7-9]. These are unlikely to be related to the structures mimicking Lewis antigens. Most $H$. pyloriinfected individuals have high titers of antibody to one of these antigenic epitope (the highly antigenic epitope). So we proposed that an LPS possessing this antigenic epitope would be a strong candidate for an antibody diagnosis of H. pylori infection [10]. Monteiro et al. [11] compared the structures between $H$. pylori LPS isolated from Asian and Western patients and found that the Asian strains showed a stronger tendency to produce type 1 blood groups. In this paper, we compared the reactivity of $H$. pylori LPS from Japanese and Western strains to the sera of $H$. pylori-positive humans.

\section{Materials and Methods}

2.1. Bacterial Strains and Preparation of LPS. Japanese $H$. pylori strains (GU2, DU1, CA2, CA4, and CA5) were isolated from biopsy specimens of lesions obtained from patients 
TABLe 1: Reactivity of LPS from Japanese and Western $H$. pylori strains against anti-Lewis antigen monoclonal antibodies and $H$. pyloripositive sera from Japanese individuals.

\begin{tabular}{|c|c|c|c|c|c|c|c|}
\hline \multirow{2}{*}{ LPS from } & \multirow{2}{*}{ Silver stain } & \multicolumn{4}{|c|}{ Reactivity of anti-Lewis antigen antibody } & \multicolumn{2}{|c|}{ Reactivity of human sera } \\
\hline & & $\mathrm{Le}^{\mathrm{x}}$ & $\mathrm{Le}^{\mathrm{y}}$ & $\mathrm{Le}^{\mathrm{a}}$ & $\mathrm{Le}^{\mathrm{b}}$ & Type A & Type $\mathrm{B}^{\mathrm{b}}$ \\
\hline \multicolumn{8}{|l|}{ Western strain } \\
\hline NCTC11637 & Rough & - & - & - & - & - & - \\
\hline Sydney & Smooth & - & + & - & - & + & - \\
\hline 26695 & Smooth & + & + & - & - & - & - \\
\hline $\mathrm{O}: 2$ & Smooth & - & - & - & - & - & - \\
\hline \multicolumn{8}{|l|}{ Japanese strain } \\
\hline GU2 & Smooth & + & - & + & - & + & - \\
\hline DU1 & Smooth & + & + & - & + & + & - \\
\hline CA2 & Smooth & - & + & - & - & - & + \\
\hline CA4 & Smooth & + & - & + & - & - & + \\
\hline CA5 & Smooth & + & + & - & - & - & + \\
\hline
\end{tabular}

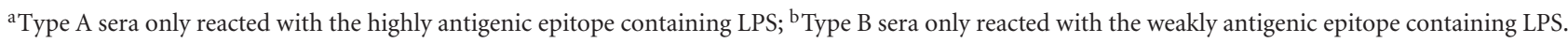

with gastric ulcer (GU), duodenal ulcer (DU), or gastric cancer (CA) in the Sapporo Medical University Hospital (Sapporo, Japan) as described previously [6]. Extraction and purification of LPS were described by Amano et al. [12]. Isolation of Western $H$. pylori strains [NCTC11637, Sydney (SS1), 26695, and O:2] and purification of LPS were as described by Monteiro et al. [4].

2.2. Human Sera. Sera from H. pylori-positive patients were donated by the Sapporo Medical University Hospital as previously described [7]. The $H$. pylori infection status of each individual was determined with the Determinar $H$. pylori antibody enzyme immunoassay kit (Kyowa Medicus, Tokyo, Japan).

2.3. Antibodies and Immunoblotting. Murine monoclonal antibodies (MAbs) against Lewis antigens used in the study were as follows: clone 73-30 [anti-Le ${ }^{\mathrm{x}}$ immunoglobulin $\mathrm{M}$ (IgM) (Seikagaku Kogyo, Tokyo, Japan)], BG8 and BG6 [anti-Le $^{\mathrm{y}}$ IgM and anti-Le ${ }^{\mathrm{b}} \mathrm{IgM}$, respectively (Signet Laboratories, Dedham, Mass, USA)], and MAB2108 [anti-Le ${ }^{\mathrm{a}}$ IgG1 (Chemicon, Temecula, Calif, USA)]. Sodium dodecyl sulfate-polyacrylamide gel electrophoresis (SDS-PAGE) and immunoblotting were performed as described previously [6]. The LPS profile on the gel was developed by silver staining as described previously [6].

\section{Results}

The molecular sizes and microheterogeneity of $H$. pylori LPS from Japanese and Western strains were compared on an SDS-PAGE gel after silver staining (Figure 1). LPS from all strains except NCTC11637 showed ladder bands, which are one of the characteristics of smooth-type LPS, in the high molecular weight area, and some bands characteristic of rough-type LPS in the low-molecular-weight area. LPS from NCTC11637 showed only one faint band in the fast

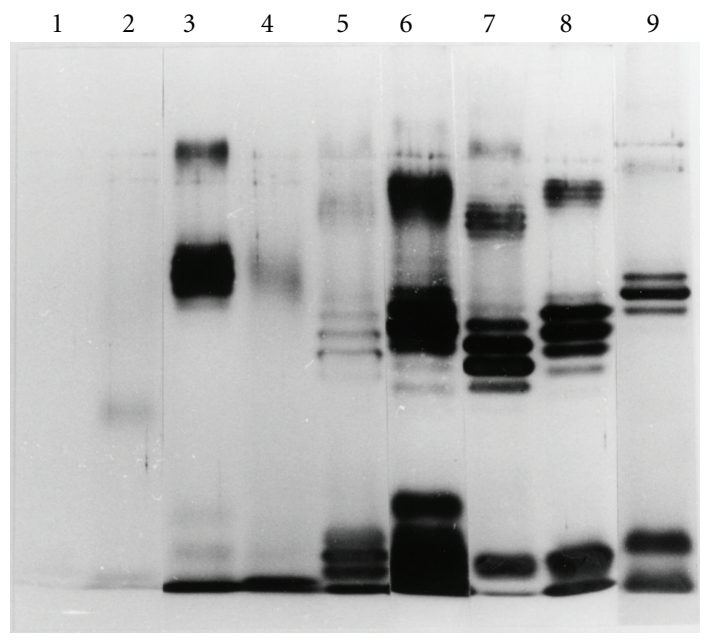

FIGURE 1: Profile of $H$. pylori LPS from Japanese and Western strains on a silver-stained SDS-PAGE gel. 1, NCTC11637-LPS; 2, Sydney strain-LPS; 3, 26695-LPS; 4, O:2-LPS, 5, GU2-LPS; 6, DU1-LPS; 7, CA2-LPS; 8, CA4-LPS; and 9, CA5-LPS.

migration zone of the gel but no ladder bands. The specificity of anti-Lewis antigen MAbs for $H$. pylori LPS was tested by immunoblotting (Table 1). LPS from the Western strains NCTC11637 and O:2 did not react with any of the MAbs. The former lost the O-polysaccharide chain, but the latter showed O-polysaccharide-containing LPS on SDS-PAGE (Figure 1). LPS from the Sydney strain reacted only with Le ${ }^{\mathrm{y}} \mathrm{MAb}$, and LPS from 26695 reacted with the $\mathrm{Le}^{\mathrm{x}}$ and $\mathrm{Le}^{\mathrm{y}}$ MAbs. Among the Japanese strains, LPS from GU2 reacted with the Le $\mathrm{e}^{\mathrm{x}}$ and $L^{a}$ MAbs; LPS from DU1 reacted with the $\mathrm{Le}^{\mathrm{x}}, \mathrm{Le}^{\mathrm{y}}$ and $\mathrm{Le}^{\mathrm{b}}$ MAbs; LPS from CA4 reacted with the $\mathrm{Le}^{\mathrm{x}}$ and Le ${ }^{\mathrm{a}}$ MAbs; LPS from CA5 reacted with the Le ${ }^{\mathrm{x}}$ and Le ${ }^{\mathrm{y}}$ MAbs; and LPS from CA2 reacted only with the Le $\mathrm{e}^{\mathrm{y}} \mathrm{MAb}$.

We examined the reactivities of sera from humans with $H$. pylori infection to $H$. pylori LPS by immunoblot 


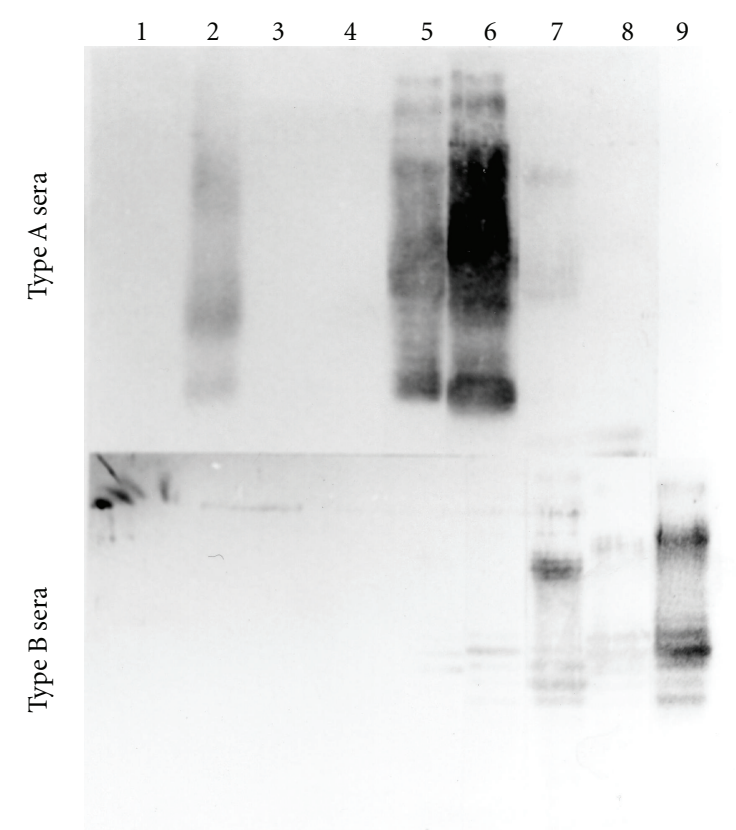

FIgURe 2: Immunoreactivities of two types of $H$. pylori-positive Japanese sera to $H$. pylori LPS from Japanese and Western strains. The LPS used are shown in Figure 1. Type A sera only reacted with LPS containing the highly antigenic epitope and Type B sera only reacted with LPS containing the weakly antigenic epitope. 1, NCTC11637-LPS; 2, Sydney strain-LPS; 3, 26695-LPS; 4, O:2-LPS, 5, GU2-LPS; 6, DU1-LPS; 7, CA2-LPS; 8, CA4-LPS; and 9, CA5LPS.

analysis. We previously proposed in [7-9] the presence of two distinct epitopes, termed the highly antigenic and the weakly antigenic epitopes, on the O-polysaccharide chains, based on data from the immunoblotting of LPS with sera from $H$. pylori-positive humans. Furthermore, we also proposed the classification of $H$. pylori-positive human sera into three groups on the basis of immunoblot reactivity to the polysaccharide region of LPS [7-9]. Type A sera only react with LPS containing the highly antigenic epitope, type B sera only react with LPS containing the weakly antigenic epitope, and type $\mathrm{C}$ sera react with both types of LPS. Among the LPS from Western strains, LPS from the Sydney strain reacted with type A sera but not type B sera (Figure 2 and Table 1). The O-polysaccharides from the NCTC11637, 26695, and O:2 strains did not react with any of the human sera, while LPS from Japanese strains reacted with either type A sera or type B sera.

\section{Discussion}

The O-polysaccharide regions of LPS are commonly used for typing gram-negative bacteria into O-serotype because of their high antigenicity, which is specific for each strain. In the case of $H$. pylori LPS, the properties of the epitopes of the polysaccharide region seem to be complex. It has been demonstrated chemically and immunogenically that the Opolysaccharide portions of $H$. pylori LPS contain structures that mimic the Lewis blood antigens $[3,4,6,13,14]$. Heneghan et al. [15] proposed that anti-Lewis antibodies were present in most patients with $H$. pylori infection and that this response is independent from the host Lewis phenotype but is related to the bacterial Lewis phenotype. However, Appelmelk et al. [16] suggested that the molecular similarity of the $H$. pylori LPS to the Lewis antigens did not promote immune evasion, nor does it lead to induction of autoantibodies. We also reported that, although high titers of antibodies to $H$. pylori LPS were found in the sera of infected patients, these antibodies were not autoreactive and were not directed against the Lewis antigens [6]. This discrepancy is interpreted to be due to differences in the Lewis antigen analogues used in the immunoanalysis.

On the other hand, our earlier studies suggest that highly and weakly antigenic epitopes reacting with human sera are unlikely to be immunogenically related to the structures mimicking the Lewis antigens [7-9]. Interestingly, the expression of the two epitopes seems to be mutually exclusive; no strain expressing both epitopes was identified, but all smooth type strains isolated in Japan expressed one or the other [7]. In this study, we observed no reaction of type A or type B Japanese sera with smooth type LPS from the Western strains 26695 and O:2, while all LPS from Japanese strains and the Sydney strain reacted with the sera belonging to the type A or B. The Sydney strain seems to be close to the Asian strains, because this strain was isolated from long-term adapted mice after they were inoculated with a fresh homogenate of gastric biopsy specimen derived from an individual in Sydney, Australia [17]. The lines of evidence indicated that the LPS in the Western strains lacked the highly antigenic and the weakly antigenic epitopes that are shared by the Japanese strains.

\section{Conclusion}

The two Western H. pylori strains shared neither the highly antigenic epitope nor weakly-antigenic epitope, which are shared by all Japanese strains, in the O-polysaccharide region of LPS.

\section{References}

[1] B. J. Marshall and J. R. Warren, "Unidentified curved bacilli in the stomach of patients with gastritis and peptic ulceration," The Lancet, vol. 1, no. 8390, pp. 1311-1315, 1984.

[2] T. L. Cover and M. J. Blaser, "Helicobacter pylori Infection, a paradigm for chronic mucosal inflammation: pathogenesis and implications for eradication and prevention," Advances in Internal Medicine, vol. 41, pp. 85-117, 1996.

[3] G. O. Aspinall, M. A. Monteiro, H. Pang, E. J. Walsh, and A. P. Moran, "Lipopolysaccharide of the Helicobacter pylori type strain NCTC 11637 (ATCC 43504): structure of the O antigen chain and core oligosaccharide regions," Biochemistry, vol. 35, no. 7, pp. 2489-2497, 1996.

[4] M. A. Monteiro, D. Rasko, D. E. Taylor, and M. B. Perry, "Glucosylated $\mathrm{N}$-acetyllactosamine $\mathrm{O}$-antigen chain in the 
lipopolysaccharide from Helicobacter pylori strain UA861," Glycobiology, vol. 8, no. 1, pp. 107-112, 1998.

[5] B. J. Appelmelk, I. Simoons-Smit, R. Negrini et al., "Potential role of molecular mimicry between Helicobacter pylori lipopolysaccharide and host Lewis blood group antigens in autoimmunity," Infection and Immunity, vol. 64, no. 6, pp. 2031-2040, 1996.

[6] K. Amano, S. Hayashi, T. Kubota, N. Fujii, and S. Yokota, "Reactivities of Lewis antigen monoclonal antibodies with the lipopolysaccharides of Helicobacter pylori strains isolated from patients with gastroduodenal diseases in Japan," Clinical and Diagnostic Laboratory Immunology, vol. 4, no. 5, pp. 540-544, 1997.

[7] S. Yokota, K. Amano, Y. Shibata et al., "Two distinct antigenic types of the polysaccharide chains of Helicobacter pylori lipopolysaccharides characterized by reactivity with sera from humans with natural infection," Infection and Immunity, vol. 68, no. 1, pp. 151-159, 2000.

[8] S. Yokota, K. Amano, S. Chiba, and N. Fujii, "Structures, biological activities and antigenic properties of Helicobacter pylori lipopolysaccharide," in Recent Research Developments in Microbiology vol. 7-Part I, S. G. Pandalai, Ed., pp. 251-267, Research Signpost, Kerala, India, 2003.

[9] S. Yokota, K. Amano, and N. Fujii, "Helicobacter pylori lipopolysaccharide as a possible pathogenic factor for gastric carcinogenesis," in Gastritis and Gastric Cancer-New Insights in Gastroprotection, Diagnosis and Treatment, P. Tonino, Ed., pp. 243-258, InTech, Rijeka, Croatia, 2011.

[10] K. Amano, S. Yokota, T. Ishioka, S. Hayashi, T. Kubota, and N. Fujii, "Utilization of proteinase K-treated cells as lipopolysaccharide antigens for the serodiagnosis of Helicobacter pylori infections," Microbiology and Immunology, vol. 42, no. 7, pp. 509-514, 1998.

[11] M. A. Monteiro, P. Y. Zheng, B. Ho et al., "Expression of histoblood group antigens by lipopolysaccharides of Helicobacter pylori strains from Asian hosts: the propensity to express type 1 blood-group antigens," Glycobiology, vol. 10, no. 7, pp. 701713,2000 .

[12] K. Amano, M. Fujita, and T. Suto, "Chemical properties of lipopolysaccharides from spotted fever group rickettsiae and their common antigenicity with lipopolysaccharides from Proteus species," Infection and Immunity, vol. 61, no. 10, pp. 4350-4355, 1993.

[13] M. A. Monteiro, K. H. N. Chan, D. A. Rasko et al., "Simultaneous expression of type 1 and type 2 Lewis blood group antigens by Helicobacter pylori lipopolysaccharides: molecular mimicry between $H$. pylori lipopolysaccharides and human gastric epithelial cell surface glycoforms," Journal of Biological Chemistry, vol. 273, no. 19, pp. 11533-11543, 1998.

[14] I. M. Simoons-Smit, B. J. Appelmelk, T. Verboom et al., "Typing of Helicobacter pylori with monoclonal antibodies against Lewis antigens in lipopolysaccharide," Journal of Clinical Microbiology, vol. 34, no. 9, pp. 2196-2200, 1996.

[15] M. A. Heneghan, C. F. McCarthy, D. Janulaityte, and A. P. Moran, "Relationship of anti-Lewis $\mathrm{x}$ and anti-Lewis $\mathrm{y}$ antibodies in serum samples from gastric cancer and chronic gastritis patients to Helicobacter pylori-mediated autoimmunity," Infection and Immunity, vol. 69, no. 8, pp. 4774-4781, 2001.

[16] B. J. Appelmelk, M. A. Monteiro, S. L. Martin, A. P. Moran, and C. M. J. E. Vandenbroucke-Grauls, "Why Helicobacter pylori has Lewis antigens," Trends in Microbiology, vol. 8, no. 12 , pp. 565-570, 2000.
[17] A. Lee, J. O’Rourke, M. C. De Ungria, B. Robertson, G. Daskalopoulos, and M. F. Dixon, "A standardized mouse model of Helicobacter pylori infection: introducing the Sydney strain," Gastroenterology, vol. 112, no. 4, pp. 1386-1397, 1997. 

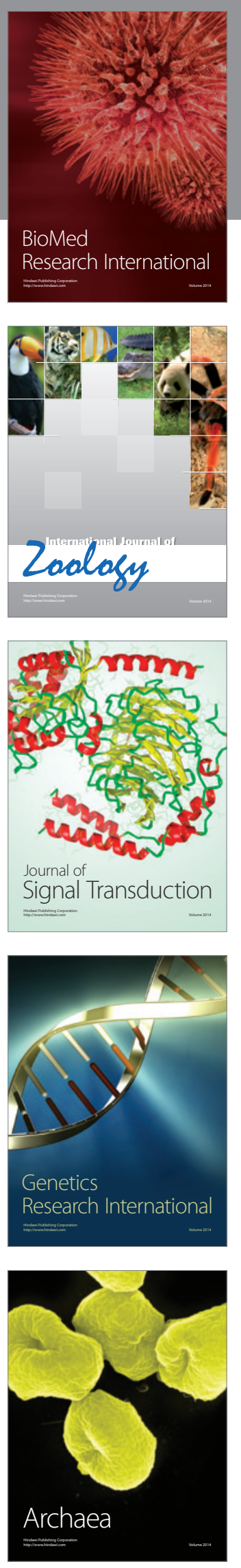
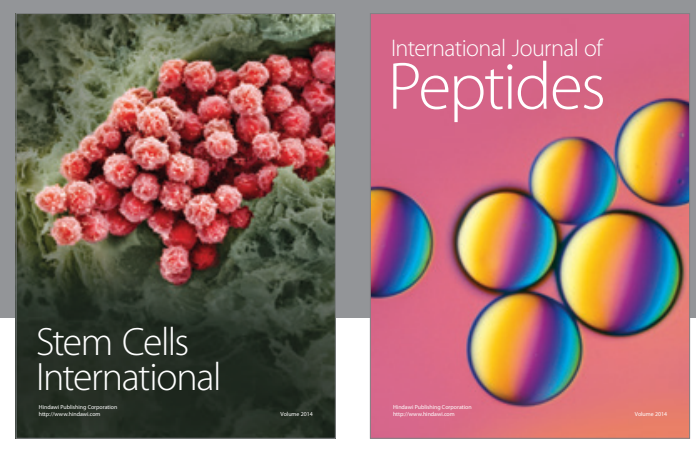

Submit your manuscripts at

http://www.hindawi.com
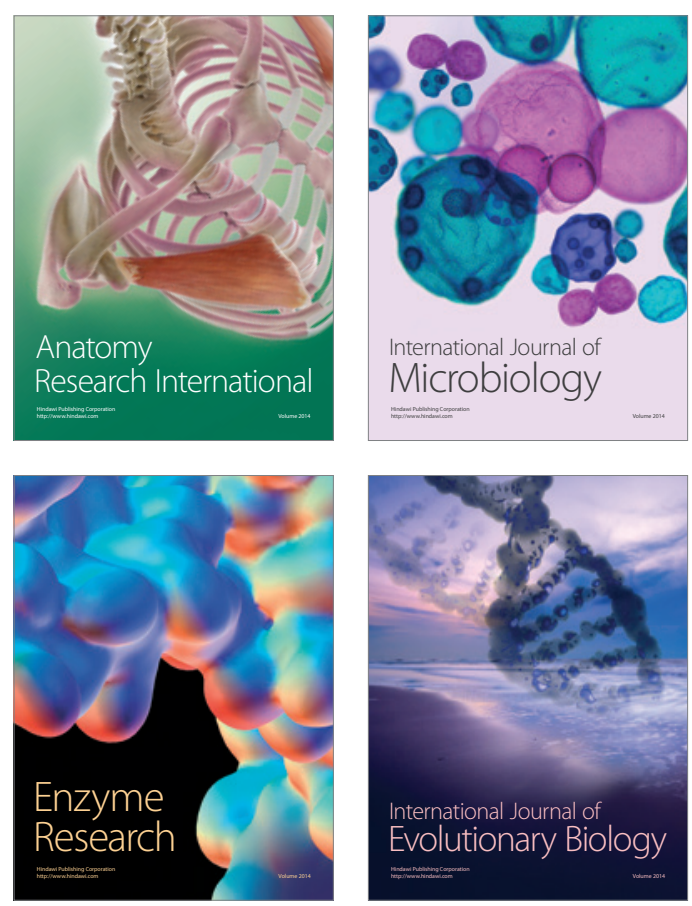
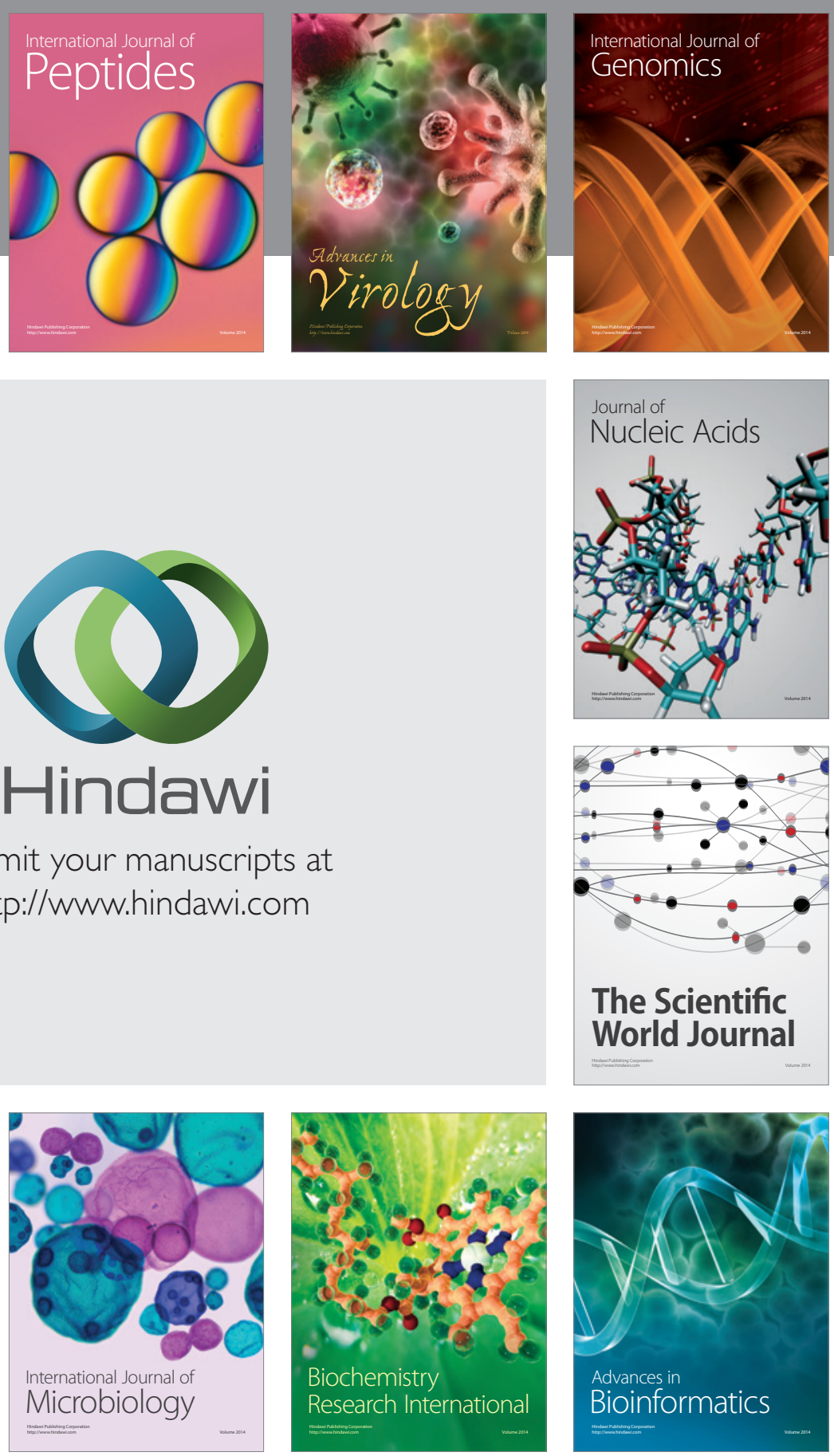

The Scientific World Journal
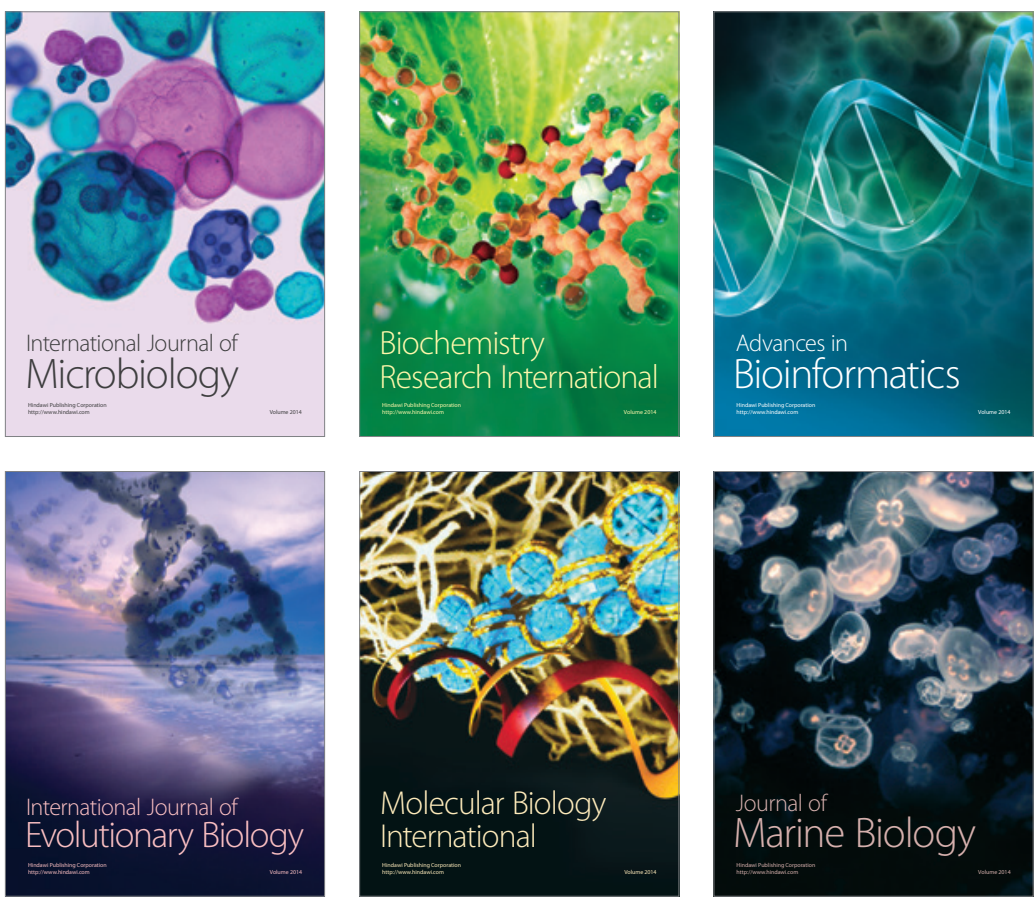